\title{
New intregral transform with Generalized Bessel-Maitland function kernel and its applications
}

\author{
Durmuş Albayrak $^{1}$, Ahmet Dernek ${ }^{1}$, Neşe Dernek ${ }^{1}$, and Faruk Ucar ${ }^{1}$ \\ ${ }^{1}$ Marmara University
}

July 12,2020

\begin{abstract}
In this paper, authors introduce the generalized Bessel-Maitland transform whose kernel is the generalized Bessel-Maitland function. New identities are obtained for special cases of the generalized Bessel-Maitland function. Using these relations, several identities are obtained for generalized Bessel-Maitland integral transform. It is shown that some special cases of them are related with the Laplace transform and the Hankel transform. Also, some examples are given as representations of the outcomes presented here.
\end{abstract}

\section{Hosted file}

addu.pdf available at https://authorea.com/users/342078/articles/468949-new-intregraltransform-with-generalized-bessel-maitland-function-kernel-and-its-applications 